\title{
Rad18 is required for functional interactions between FANCD2, BRCA2, and Rad51 to repair DNA topoisomerase 1-poisons induced lesions and promote fork recovery
}

\author{
Kaushlendra Tripathi ${ }^{1}$, Chinnadurai Mani ${ }^{1}$, David W Clark $^{1}$, Komaraiah Palle ${ }^{1}$ \\ ${ }^{1}$ Department of Oncologic Sciences, Mitchell Cancer Institute, University of South Alabama, Mobile, Alabama, 36604, USA \\ Correspondence to: Komaraiah Palle, e-mail: kpalle@health.southalabama.edu \\ Keywords: Rad18, FANCD2, Rad51, BRCA2, DNA topoisomerase 1
}

Received: October 27, $2015 \quad$ Accepted: January 27, $2016 \quad$ Published: February 08,2016

\section{ABSTRACT}

Camptothecin (CPT) and its analogues are chemotherapeutic agents that covalently and reversibly link DNA Topoisomerase $I$ to its nicked DNA intermediate eliciting the formation of DNA double strand breaks (DSB) during replication. The repair of these DSB involves multiple DNA damage response and repair proteins. Here we demonstrate that CPT-induced DNA damage promotes functional interactions between BRCA2, FANCD2, Rad18, and Rad51 to repair the replication-associated DSB through homologous recombination (HR). Loss of any of these proteins leads to equal disruption of HR repair, causes chromosomal aberrations and sensitizes cells to CPT. Rad18 appears to function upstream in this repair pathway as its downregulation prevents activation of FANCD2, diminishes BRCA2 and Rad51 protein levels, formation of nuclear foci of all three proteins and recovery of stalled or collapsed replication forks in response to CPT. Taken together this work further elucidates the complex interplay of DNA repair proteins in the repair of replication-associated DSB.

\section{INTRODUCTION}

DNA topoisomerase 1 (Top1) is an essential enzyme in higher eukaryotes, which resolves topological barriers during most critical cellular processes involving DNA, including replication, transcription, recombination and repair [1-4]. Top1 modulates DNA topology by introducing a transient single strand break through active site tyrosine (Tyr723) forming a covalent 3'-phosphotyrosyl bond with DNA, which is called Top1DNA covalent or cleavage complex (Top1cc) [5-8]. The formation of Top1cc is rapidly followed by a second transesterification reaction to reseal the broken strand and to maintain DNA integrity. Topological strain in the DNA drives the broken strand to rotate around the intact strand thereby relieving the extent of supercoiling during the period between the cleavage and religation, allowing transcription or replication to proceed [9]. In normal conditions, the covalently bound Top $1 \mathrm{cc}$ is transient and mostly undetectable because religation is much faster than cleavage in Top1 catalysis $[3,4]$. However, anticancer agents such as camptothecin (CPT) and its clinical analogues (ex. topotecan and irinotecan) intercalate into the Top1 generated DNA nick and inhibit the religation of scissile strand, which greatly prolongs the half-life of Top1cc $[9,10]$. These CPT stabilized Top1cc are not known to be cytotoxic by themselves and are readily reversible after removal of the drug. However, prolonged stabilization of Top1cc can create multiple problems. Firstly, failure to relieve supercoiling generated by such processes as transcription and replication can lead to replication stress by creating torsional strain within the DNA $[9,11,12]$. Furthermore, collision between an active replication fork and the Top1cc is capable of generating DNA double strand breaks (DSB) which can introduce mutations or lead to cell death [13-15]. Anticancer agents, such as DNA Top1 poisons as well as others that result in stalled replication forks (hydroxyurea, gemcitabine, and others) are effective as they lead to high levels of DSB in rapidly replicating cancer cells as opposed to quiescent terminally differentiated normal cells $[14,16$, 17]. Studies in yeast and human cancer cells identified homologous recombination (HR) as the predominant DNA repair pathway involved in repairing CPT-induced DSB [18-23]. Correspondingly, deficiencies in proteins involved in HR (ex. Rad51) as well as associated proteins such as Rad18, members of the Fanconi anemia (FA) family and the breast cancer associated (BRCA1, BRCA2) proteins sensitize cells to CPT [24-28]. 
Rad18 belongs to zinc and RING finger family of E3 ubiquitin ligases and its functions are well studied in post-replication repair pathway also known as translesion synthesis (TLS) [29-33]. Rad18 regulates TLS by monoubiquitinating PCNA, thereby triggering a polymerase switch allowing bypass of the bulky adducttype of DNA lesion [31, 32]. Thus cells defective in $\operatorname{Rad} 18$ fail to faithfully replicate DNA over a variety of mutagenic adducts and exhibit hypersensitivity to the presence of these lesions [32]. Moreover, Rad18 has also been to shown to play an important role in repair of DSB by directly binding to Rad51C, a paralog of Rad51 and localize it to sites of DSB to promote HR [26]. We and others have previously shown that Rad18 regulates the FA pathway in response to fork stalling lesions induced by several agents, including CPT [12, 34-37]. These studies demonstrated that Rad18 E3 ligase activity is important for efficient FANCD2 monoubiquitination, as well as its CPT-induced nuclear foci formation and cell survival by timely repair of replication-coupled DSB [12, 37, 38]. The FA proteins (FANCs) associate with replication forks and have been implicated in repair of fork stalling lesions by $\mathrm{HR}$ in association with BRCA proteins [3944]. BRCA2 is also known as FANCD1 and is a member of both families of proteins [40], and recently BRCA1 was given the alternate name FANCS as mutations in BRCA1 are capable of causing FA [41]. An epistatic relationship between FA and BRCA genes has been suggested in repair of replication fork stalling lesions by HR; however, regulation of FA-BRCA proteins and their molecular interactions are rather complex and not well known [42-44]. In this study we focused on the role of Rad18-mediated activation of FANCD2 and its functional relationship with BRCA2 and Rad51 proteins in repair of CPT-induced lesions. Our data show that downregulation of Rad18 or FANCD2 leads to decreased BRCA2 and Rad51 foci formation and co-localization in response to CPT. Consistently, either single gene downregulation or co-depletion with Rad18 resulted in similar levels of sensitivity to CPT and increase in gross chromosomal aberrations (CA). Moreover, Rad18, FANCD2, BRCA2 and Rad51 co-immunoprecipitated and depletion of Rad18 suppressed this interaction, suggesting these proteins work in a common repair pathway to promote accurate repair of CPT-induced replication-coupled DSB.

\section{RESULTS}

\section{Rad18 is required for proper FANCD2, BRCA2, and Rad51 foci formation in response to CPT- induced DSB}

Stabilization of Top1cc by CPT and its clinical analogues induces replication stress and replicationcoupled DSB. We recently reported that E3 ligase activity of Rad18 is important for FA pathway activation to efficiently repair CPT-induced DSB and for cell survival [12]. Rad18 has also been shown to interact with Rad51C and promote its recruitment to sites of DSB in an E3 ligase-independent manner [26]. However, several studies established functional interactions and epistatic relationship between FANCD2, BRCA2 and Rad51 in stabilization of stalled replication forks and repair of collapsed forks by HR [41, 44-46]. $\operatorname{Rad} 18$ is important for efficient activation of the FA pathway; however, the functional interplay between Rad18 status and FANCD2, BRCA2 and Rad51 proteins in repair of replicationassociated DNA lesions is not known. To examine this, we transiently downregulated these genes and examined their influence on each other in response to Top1 poison CPT.

As expected, exposure of H1299 cells to CPT elicited a robust DNA damage response by inducing Rad18, FANCD2, BRCA2 and Rad51 nuclear foci formation (Figure $1 \mathrm{~A}$ and 1B). Cells treated with the vehicle (DMSO) alone exhibited little or no detectable foci of these proteins (data not shown). The FA pathway was activated in response to CPT as evidenced by the monoubiquitination of FANCD2 (Figure 2A) and its nuclear localization (Supplementary Figure S1C). Consistently, CPT exposure induced replication stress and associated DSB as indicated by $\gamma \mathrm{H} 2 \mathrm{AX}$ and FANCD2 foci and their co-localization with EdU (5-ethynyl-2'-deoxyuridine) (Supplementary Figure S1A) and induction of 53BP1 foci (Supplementary Figure S1B). As reported previously, Rad18 downregulation attenuated CPTinduced monubiquitination of FANCD2 (Figure 2A and Supplementary Figures S2A and S2B), its nuclear localization (Supplementary Figure S1C) and led to an approximately $50 \%$ decrease in its foci formation (Figure 1A and 1C). However, FANCD2 depletion did not cause a significant decrease in Rad18 nuclear foci formation (Figure 1A) in response to CPT. Moreover, either downregulation of $\operatorname{Rad} 18$ or FANCD2 in these cells, substantially decreased CPT-induced BRCA2 $(\approx 50 \%)$, and $\operatorname{Rad} 51 \quad(\approx 50 \%)$ foci and their colocalization (Figure 1A, 1B, 1C and Supplementary Figure S4B and S4D). Similarly, knocking down either $\operatorname{Rad} 18$ or FANCD2 also resulted in decrease of basal and CPT-induced levels of BRCA2 and Rad51 proteins compared to their respective controls (Figure $2 \mathrm{~A}$ and $2 \mathrm{~B}$ ). However, further studies are needed to fully elucidate the molecular basis for this decrease in BRCA2 and Rad51 protein level. To rule out that these effects are cell line specific or off-target effects of siRNAs, these results were further confirmed in A2780 (ovarian cancer) cells (Supplementary Figure S2A) and using an siRNA targeting 3'-UTR region of Rad18 transcript (Supplementary Figure S2B). Furthermore, consistent with previous studies [12, 29], E3 ubiquitin ligase activity of Rad18 is important for the FANCD2 foci formation (Supplementary Figure S3A), and its 
nuclear localization (Supplementary Figure S3B). Collectively, these results indicate a functional relationship between these four proteins in response to CPT-induced DNA damage. Recently, FANCD2 has been shown to form a functional complex with Rad18 and Rad51 and rescue stalled replication forks resulting from dNTP depletion due to HU treatment $[45,46]$. FANCD2 also directly interacts with BRCA2, and this functional interaction is necessary for proper BRCA2 foci formation and efficient repair of DSB by HR [43, 47, 48]. Based on these observations, our data indicate that $\operatorname{Rad} 18$ may act upstream to other three proteins and regulate this functional interaction by promoting FANCD2 monoubiquitination and its nuclear localization in response CPT-induced fork-stalling lesions.

\section{BRCA2 and Rad51 act downstream of Rad18 and FANCD2 in response to CPT-induced DSB}

To determine whether BRCA2 and $\operatorname{Rad} 51$ have any reciprocal effect on $\operatorname{Rad} 18$ and FANCD2 foci formation and stability, H1299 cells were transfected with BRCA2 and Rad51 siRNAs and their responses to CPT were monitored. Transient depletion of BRCA2 did not alter FANCD2 monoubiquitination (Figures 3, 4A and Supplementary Figure S4A) or Rad18 protein levels or their foci formation in response to CPT (Figure 4A,
A

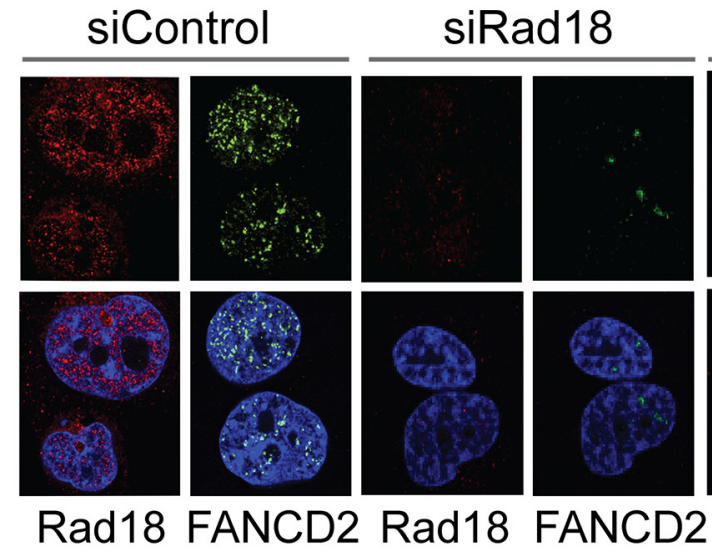

SiFANCD2

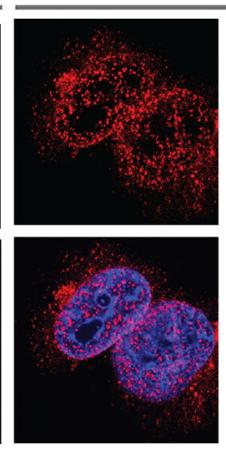

Rad18 FANCD2
DAPI

Merged

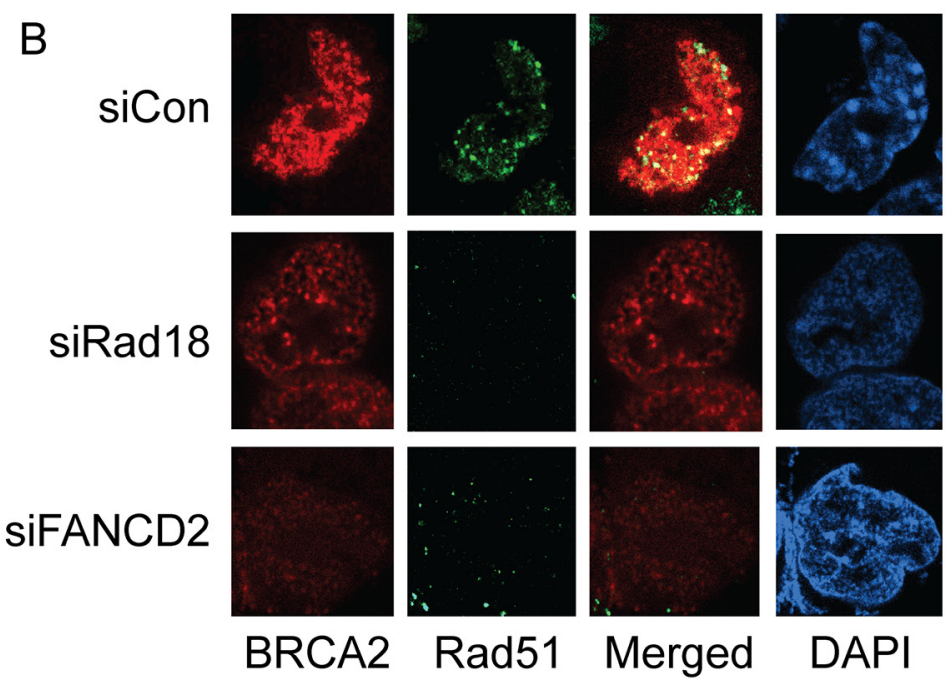

C

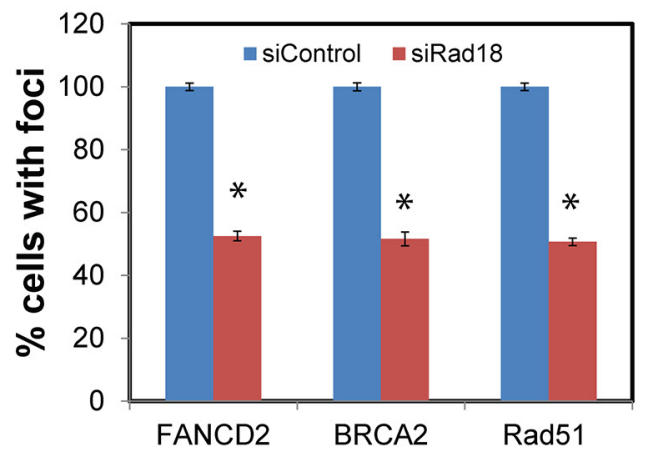

Figure 1: Effect of Rad18 or FANCD2 depletion on Rad18, FANCD2, BRCA2 and Rad51 foci formation in response to CPT. H1299 cells were transfected with siRNA directed against either Rad18 or FANCD2 or a control siRNAs and were exposed to $500 \mathrm{nM}$ CPT for 2 hours, fixed and labeled with the indicated antibodies and stained with fluorophore-labeled secondary antibodies and nuclei were stained with DAPI. The immunofluorescence images were acquired using Nikon Ti eclipse confocal microscope at 100X. Cells treated with DMSO did not demonstrate any significant, detectable foci (data not shown). A. The effects of knocking down of Rad18 on foci formation of FANCD2, and Rad18 foci formation in FANCD2-knockdown cells in response to CPT. B. The effects of depleting either Rad18 or FANCD2 on foci formation of BRCA2 and Rad51 in response to CPT. The merged panel shows the extent of co-localization between BRCA2 and Rad51 under these conditions. C. Graph showing the normalized mean of three independent experiments with bars representing \pm S.D. * Denotes statistical significance compared to their respective controls $(\mathrm{P}<0.05)$. 
Supplementary Figure S4C and data not shown). However, BRCA2 knockdown resulted in decrease of $\operatorname{Rad} 51$ protein (Figure 4A) and its CPT-induced foci formation (Figure 3 and Supplementary Figure S4B). These findings suggest that BRCA2 may act downstream of both $\operatorname{Rad} 18$ and FANCD2 in CPT-induced DNA damage response.

Interestingly, Rad51 depletion had no significant impact on either the protein levels (Figure 4B) or the CPTinduced foci formation of Rad18, FANCD2, or BRCA2 (Figures 3, 4B, and Supplementary Figures S4A, S4C, $\mathrm{S} 4 \mathrm{D}$, and S5). Furthermore, Rad51 does not seem to alter co-localization of the other three proteins (Figure 3, merged panels and Figure 5B). Conversely, $\operatorname{Rad} 51$ protein levels and its foci formation were diminished by depletion of Rad18, FANCD2 and BRCA2 (Figures 1A, 1B, 2A, 2B, 3, 4A and Supplementary Figure S4B). These results suggests that Rad51 acts downstream of Rad18, FANCD2 and BRCA2 in response to CPT and its levels or stability depends on the status of the other three proteins.

\section{Rad18, FANCD2, BRCA2, and Rad51 interact and co-localize at the CPT-induced DSB}

Rad18, FANCD2, BRCA2 and Rad51 all are known to be involved in DSB repair by HR. Several studies demonstrated the molecular interactions between

A
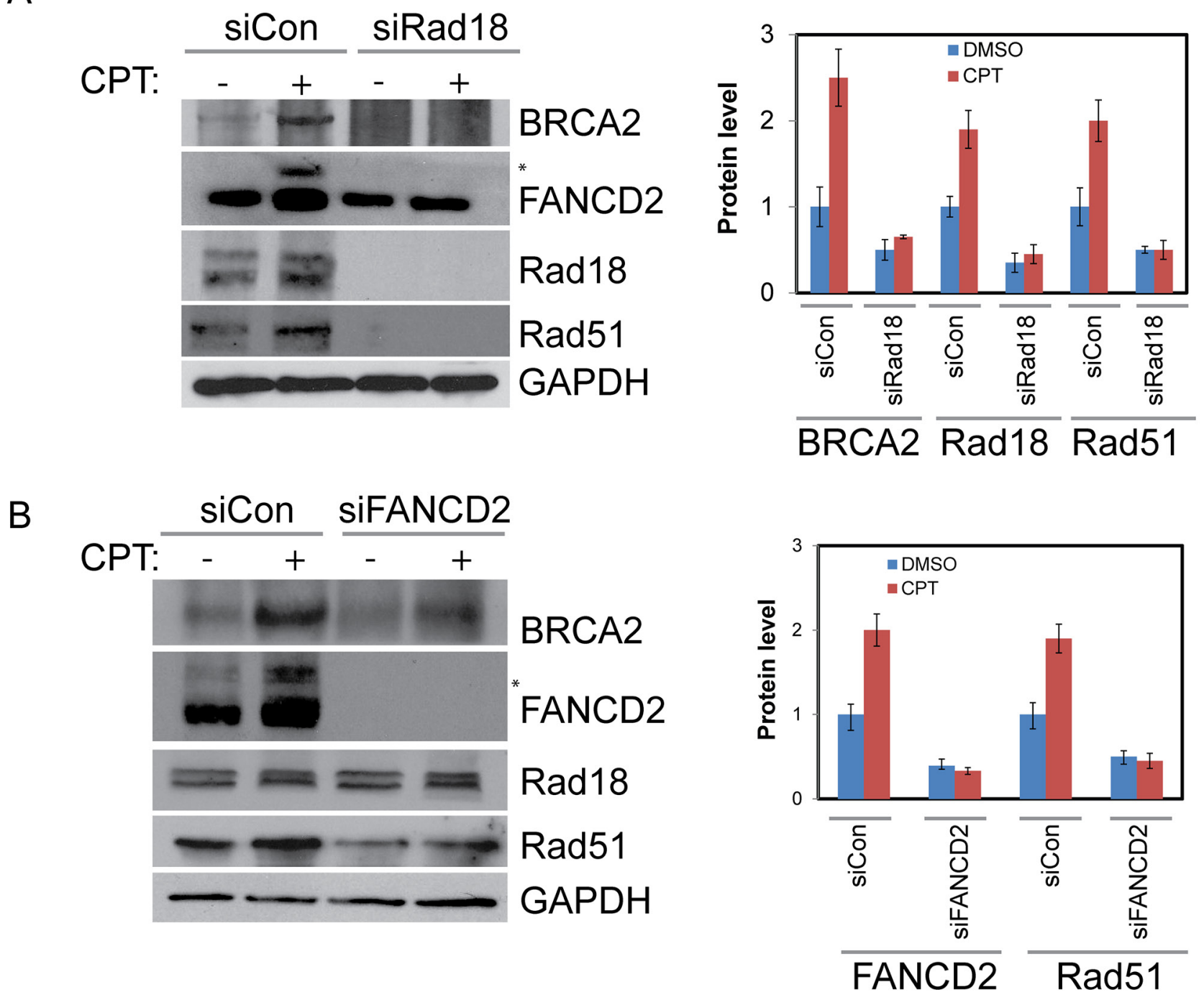

Figure 2: Rad18 is important for proper activation of FANCD2 and both Rad18 and FANCD2 are important for the stability of Rad51 and BRCA2. H1299 cells were depleted of Rad18 or FANCD2 by siRNAs or treated with control siRNAs for 48 hours. Cells were then treated with either DMSO or $500 \mathrm{nM} \mathrm{CPT} \mathrm{for} 2$ hours and proteins were harvested. A. Western blot shows the impact of Rad18 down-regulation on monoubiquitination status of FANCD2 as indicated by (*asterisk), and levels of BRCA2 and Rad51 proteins. Densitometry analysis of blots from multiple experiments presented in histogram. B. Western blot showing the effects of FANCD2 knockdown on Rad18, Rad51, and BRCA2 protein levels basally and in response to CPT, densitometric analysis of blots from multiple experiments presented in histogram and the error bars indicate \pm S.D. 
some of these proteins and their interdependence [4046]. Based on these observations, our data suggests that these proteins may act together in the repair of CPT-induced DNA lesions and may promote stability, activation, and/or ability to form DNA repair foci of each other. Therefore, it is important to determine if these proteins form a functional complex or interact, directly or indirectly, in response to DNA damage. To this end, co-immunoprecipitation assays were performed in cells treated with CPT. The protein levels were normalized for each immunoprecipitation reaction (Figure 5A, bottom panel) and immunoglobulin $\mathrm{G}$
(IgG) was used as a negative control. As expected, Rad18, FANCD2, BRCA2 and Rad51 all coimmunoprecipitated each other (Figure 5A, top panel), suggesting that these proteins all interact directly or indirectly to form a functional complex in response to CPT. To further confirm whether $\operatorname{Rad} 18$ is required for these interactions, the ability of FANCD2 to immunoprecipitate Rad51 and BRCA2 in cells treated with siRad18 was tested. When Rad18 was depleted, FANCD2 failed to immunoprecipitate BRCA2 and Rad51 proteins (Supplementary Figure S6), suggesting $\operatorname{Rad} 18$ is important for these functional interactions.

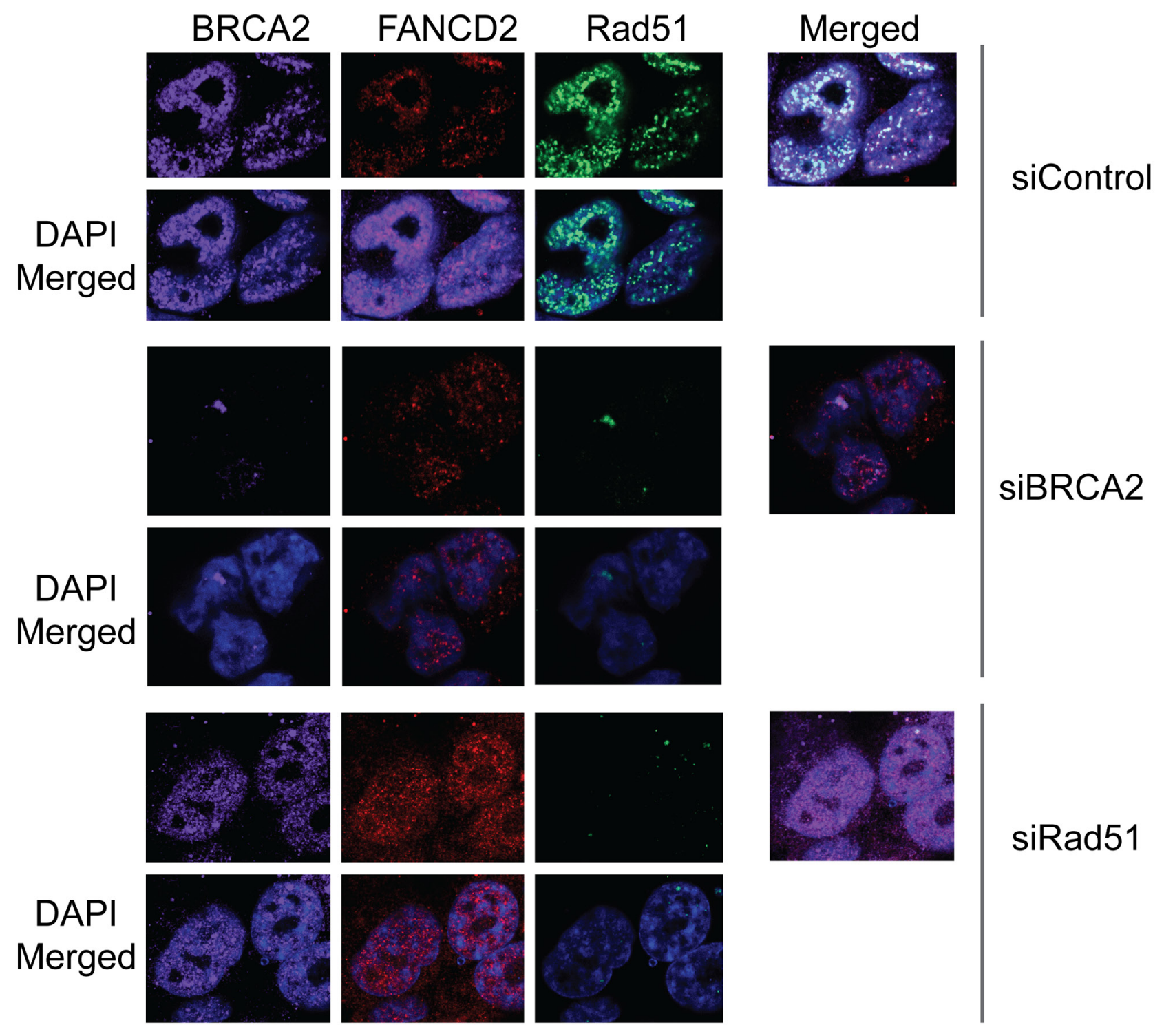

Figure 3: Downregulation of Rad51 or BRCA2 does not significantly effect on CPT-induced foci formation of Rad18 and FANCD2. H1299 cells were treated with siRNAs directed against either Rad51 or BRCA2 or control siRNAs and were exposed to $500 \mathrm{nM} \mathrm{CPT}$ for 2 hours, fixed and labeled with the indicated antibodies and stained with fluorophore-labeled secondary antibodies and visualized by immunofluorescence microscopy. Nuclei were stained with DAPI. Cells treated with DMSO did not demonstrate any significant, detectable foci and are not shown. (A) The formation of BRCA2, FANCD2 and Rad51 foci in response to CPT-induced DSB in cells depleted in either BRCA2 or Rad51 compared to control siRNA-treated cells. The extent of co-localization of the three proteins is shown in their respective right panels labeled as "merged". 
These differences could be due to the decreased levels of the proteins or that Rad18 is necessary for the interactions between FANCD2, BRCA2 and Rad51. However, further work is needed to determine the molecular basis of these interactions and if all of these proteins are part of a large DNA repair complex. Interestingly, in these immunoprecipitations, a significant amount of the FANCD2 pulled down was the monoubiquitinated form in control cells (Supplementary Figure S6, indicated by asterisk), whereas in Rad18deficient cells it is mostly unmodified FANCD2.
Rad18, FANCD2, BRCA2 and Rad51 work in a common pathway to repair CPT-induced DSB by HR

Replication-coupled DSB, such as those induced by CPT, are predominantly repaired by HR-mediated repair [18]. Deficiencies in Rad18, FANCD2, BRCA2, and Rad51 are all known to sensitize tumor cells to CPT. Data shown above suggest that BRCA2, FANCD2, Rad18, and Rad51 functionally interact and may enhance the repair of DNA lesions induced by CPT by HR. To further examine
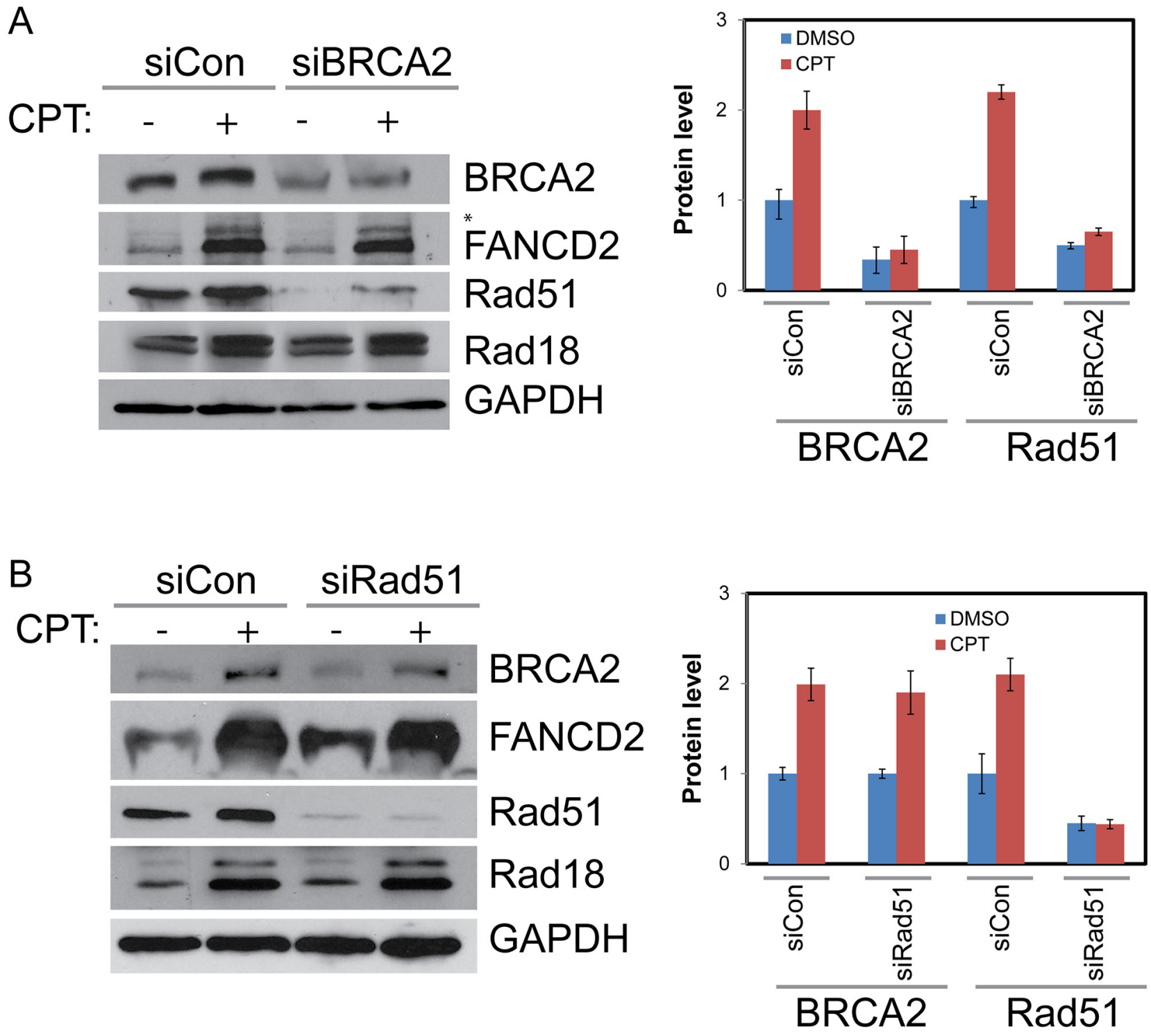

Figure 4: BRCA2 increases stability of Rad51 in CPT-treated cells but Rad51 has no detectable impact on BRCA2, Rad18 or Rad51. A. H1299 cells were depleted of BRCA2 by siRNA or treated with a control siRNA for 48 hours. They were then treated with vehicle or $500 \mathrm{nM} \mathrm{CPT}$ for 2 hours and proteins were harvested. Western blot shows downregulation of BRCA2 diminishes Rad51 protein levels basally and in response to CPT treatment but has little impact on Rad18 or FANCD2. B. Rad51 knockdown has no significant impact on BRCA2, Rad18 protein levels and monoubiquitination of FANCD2 (as indicated by *asterisk) basally and in response to CPT. Histogram in the right panels shows the densitometric analysis of blots from multiple experiments and the error bars indicates \pm S.D.of. 
A

IPs: IgG R18 IgG FD2 IgG BC2

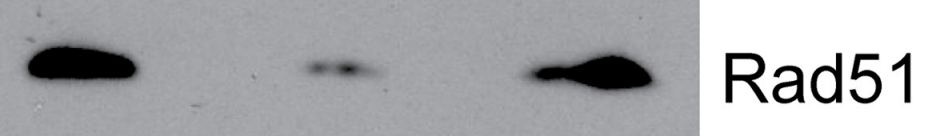

IPS: IgG R18 IgG BC2 IgG R51

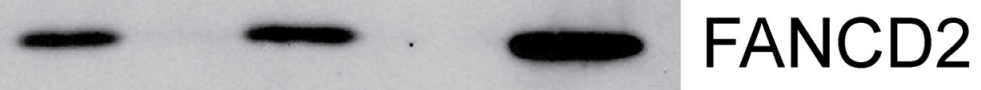

IPS: IgG FD2 IgG R51 lgG BC2

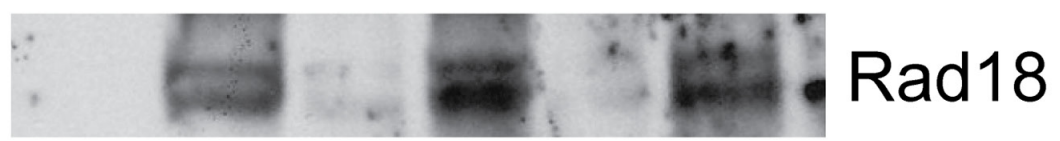

\section{Inputs}

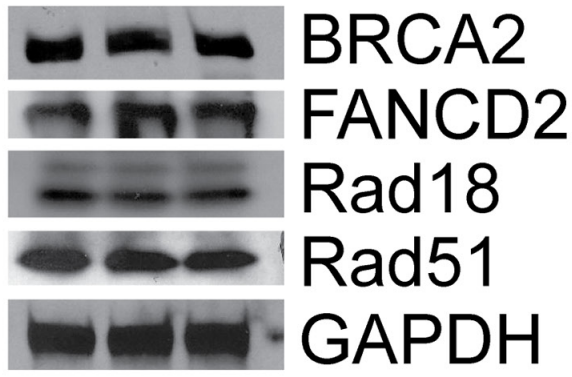

B
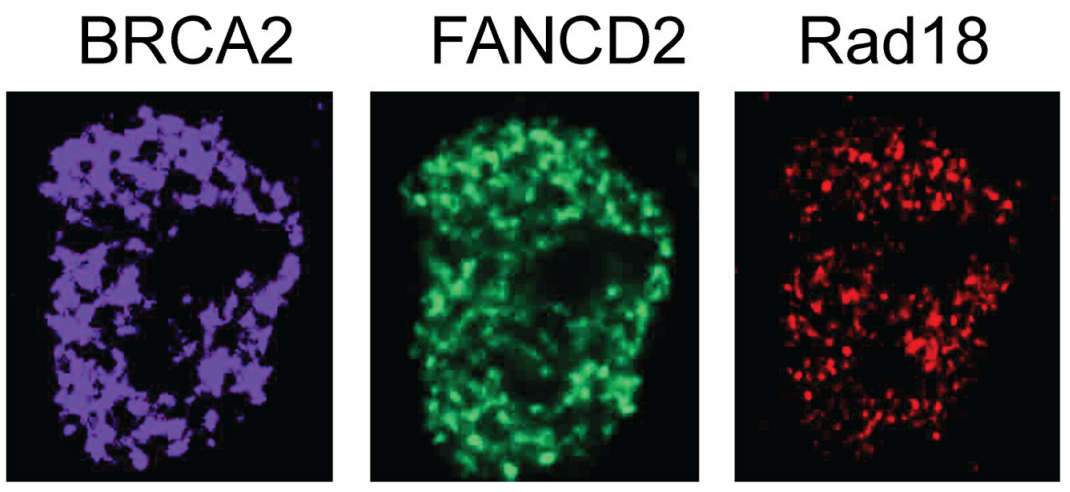

Merged
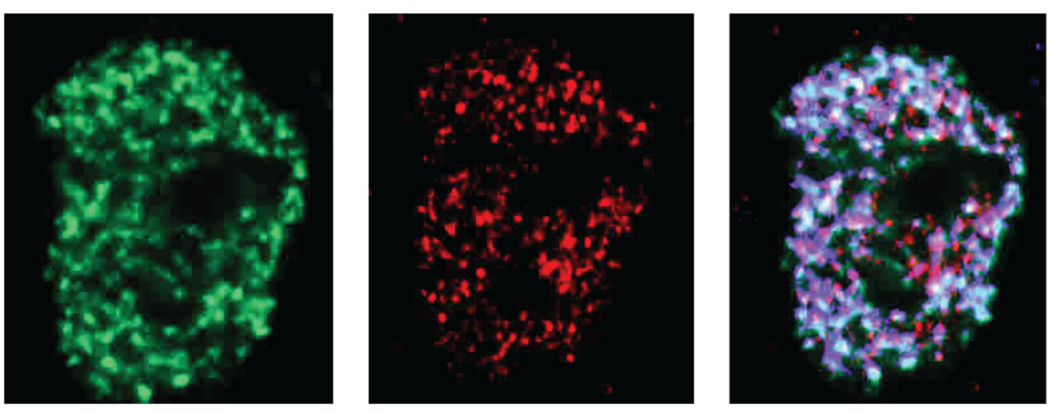

Figure 5: Rad18, FANCD2, BRCA2 and Rad51 co-immunoprecipitates and co-localizes in response to CPT. H1299 cells were treated with $500 \mathrm{nM} \mathrm{CPT}$ for 2 hours and proteins were isolated and immunoprecipitated with antibodies against Rad51, FANCD2 BRCA2 and Rad18. Immunoglobulin G (IgG) used as negative control. A. Each immunoprecipitated sample was tested for the presence of the other 3 proteins by Western blots and the bottom panel shows the equal levels of proteins in input protein samples. B. Rad51 was knocked down in $\mathrm{H} 1299$ cells and treated with $500 \mathrm{nM}$ CPT for 2 hours and the co-localization of the remaining three proteins was visualized by immunofluorescences. The images show that Rad51 is not required for the co-localization of the Rad18, FANCD2 and $\mathrm{BRCA} 2$ proteins in response to $\mathrm{CPT}$. 
the coordination of these proteins in the HR process, each gene was knocked down singly or in combination with $\operatorname{Rad} 18$ and assessed for sensitivity to CPT by clonogenic survival assay. Depletion of Rad18, FANCD2, BRCA2 and Rad51 all resulted in similar levels of CPT hypersensitivity compared to control cells (Figure 6A and 6B). Additionally, when BRCA2, FANCD2 and Rad51 were knocked down in combination with $\operatorname{Rad} 18$, there was little change from the single knockdown samples. To rule out that these results are specific to one cell line, these results were further confirmed using Rad18 wild-type and Rad18-null HCT116 cells transfected with siRNAs for FANCD2, Rad51 and BRCA2 (Supplementary Figure S7A and S7B). In these cells, there was no significant difference between the $\operatorname{Rad} 18 \mathrm{KO}$ cells and those transiently depleted for any of the other proteins. These results further indicate that Rad18, FANCD2, BRCA2, and Rad51 function together in the same pathway to repair CPT-induced, replication-coupled DSB.

To further assess the contributions of each of these proteins in repair of DSB by HR, DR-GFP reporter assays were performed utilizing site specific endonuclease I-SceI that generates a DSB in a GFP reporter. Background GFP expression is very low as determined by an I-SceI negative control (Figure 7A, first panel). Cells transfected with control siRNA exhibited GFP expression and represent the normal HR repair capability (Figure 7A, second panel). Depletion of Rad18, FANCD2, BRCA2 and Rad51 each exhibited similar decrease (all around $50 \%$ of control) in the HR efficiency in these cells (Figure 7B and 7C). Therefore, BRCA2, FANCD2, Rad18 and Rad51 each appear to contribute to repair and function in the HR pathway.

\section{Functional interaction between Rad18, FANCD2, BRCA2 and Rad51 suppresses error prone pathways in repair of CPT-induced DNA lesions}

In S-phase, Rad18, FANCD2, BRCA2, and Rad51 were all found to play important roles in maintaining stability of stalled replication forks and timely repair of collapsed forks by error free HR to maintain genome integrity [45-48]. Thus, deficiency in these genes compromises HR efficiency, which could lead to cell death or repair through error prone recombination mechanisms, such as non-homologous end joining (NHEJ), increasing the frequency of chromosomal aberrations (CA). Deficiencies in FA-BRCA-Rad51 tumor suppressor group have been shown to induce gross CA in response to a variety of genotoxins [49-51]. To assess whether Rad18 deficiency also enhances CA frequency, we downregulated these genes in H1299 cells alone or in combination with Rad18. Metaphase spreads were scored for CA in cells treated with either vehicle (DMSO) or CPT. Figure 8A shows a significant increase in the frequency of spontaneous CA formation in FANCD2- and BRCA2-depleted cells compared to controls. Upon exposure to CPT, cells deficient in any of the single genes or combined with Rad18 knockdown showed statistically significant increases in the CA frequency compared to control cells. Furthermore, the magnitudes of CA frequency in each of the knockdowns were similar. Examples of the CA produced by CPT are shown in Figure $8 \mathrm{~B}$ and Supplementary Figure S8. Deficiency in FA genes has been shown to induce radial formation upon exposure to genotoxins [51]. Interestingly, a significant increase in spontaneous and CPT-induced radial chromosomes was observed in Rad18-deficient cells (Figure 8C). Although a similar frequency of gross CA was observed in single gene knockdowns and co-depletions with $\operatorname{Rad} 18$, the frequency of radial chromosomes was significantly higher in FANCD2- or BRCA2-deficient cells. This could be due to independent functions of these proteins in either interactions with other DDR proteins or their role in suppression of error-prone DNA repair pathways such as NHEJ [52-56].

\section{Rad18 stabilizes replication forks in CPT-treated cells}

Several studies demonstrated important roles for FA-BRCA proteins in maintaining replication fork stability and restart of the stalled or collapsed forks in response to genotoxic stress [44]. To analyze the role of $\operatorname{Rad} 18$ in maintaining replication fork stability and recovery, DNA fiber assays were performed as described [44, 63, 64]. To measure the fork velocity in H1299 cells transfected with control or Rad18 siRNAs, cells were labeled with CldU for thirty minutes followed by labelling with IdU for thirty minutes in the presence or absence of CPT (Figure 9A). Transient depletion of $\operatorname{Rad} 18$ does not significantly alter fork velocity (siControl and siRad18 cells were 1.04 and $1.0 \mathrm{~Kb} / \mathrm{min}$, respectively). However, when cells were exposed to CPT, those cells deficient in Rad18 exhibited substantially decreased fork velocity (siControl: 0.59 and siRad18: $0.38 \mathrm{~Kb} / \mathrm{min}$ ) (Figure 9B-9C). Furthermore, to determine if $\operatorname{Rad} 18$ also affects the ability of replication forks to restart after CPT-induced fork stalling, cells were treated with CPT and labeled with nucleoside analogues as shown in Figure 9D. Interestingly, restart or reversal of stalled fork in the presence of CPT in Rad18-depleted cells was approximately half that of Rad18-proficient cells (Figure 9E). Taken together this data demonstrates that while Rad18 is not necessary for DNA replication to proceed under normal conditions, it is vital for maintaining the stability of replication forks when they encounter a block, such as CPT-generated Top1cc, likely through activation and recruitment of FA pathway and Rad51 and associated proteins. 
A

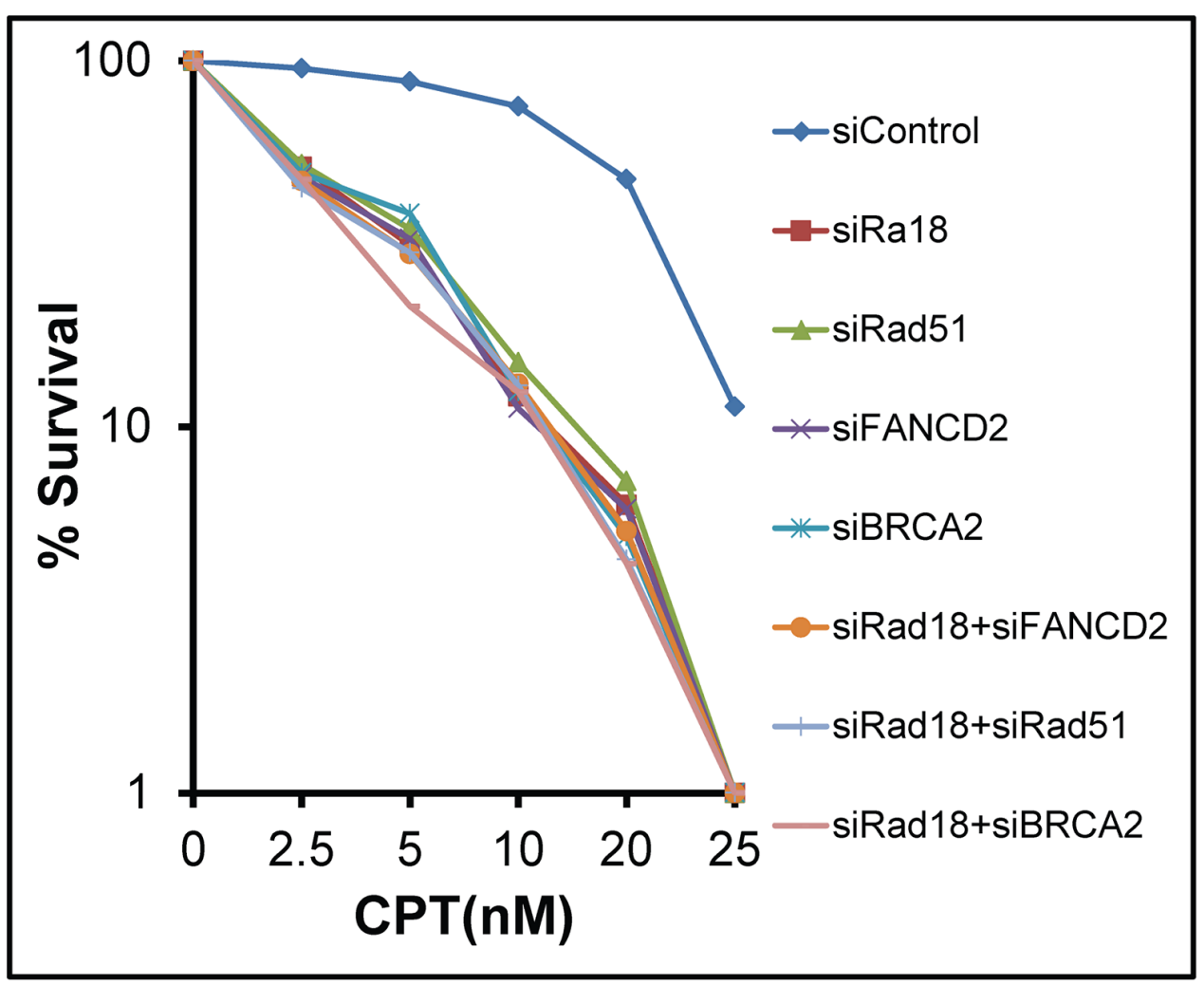

B
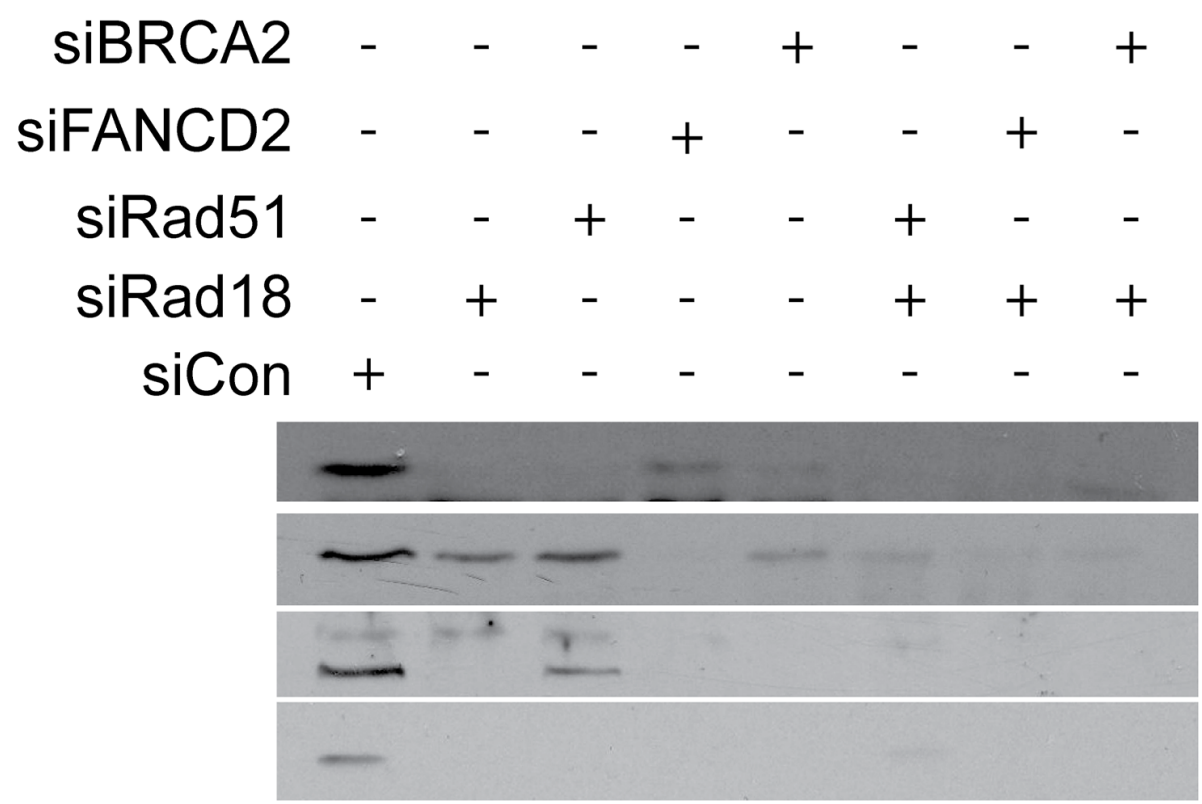

BRCA2

FANCD2

Rad18

Rad51

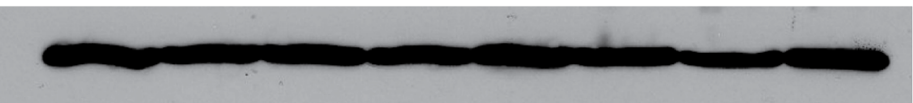

Figure 6: Depletion of Rad18, FANCD2, BRCA2 or Rad51 singly or in combination with Rad18 sensitizes cells to CPT to similar extent. H1299 cells were treated with siRNA to knock-down Rad18, FANCD2, BRCA2 and Rad51 alone or in combination with Rad18. These cells were treated overnight with the indicated concentration of CPT or DMSO and surviving cells were allowed to form colonies. Colonies containing at least 25 cells were counted and plotted (top panel). The clonogenic survival assays were performed in triplicates and graph represents means of three independent experiments with error bars representing the \pm S.D. The bottom panel shows a representative Western blot to confirm depletion of the targeted proteins. 


\section{DISCUSSION}

Camptothecin and its chemotherapeutic analogues cause replication-associated DNA strand breaks that are primarily repaired by homologous recombination $[18$, 20]. This could be due to the nature of the DNA lesions generated by the Top 1 inhibitors, and/or the availability of homologous chromosomes in S and G2 phases. Indeed, repair of these breaks by NHEJ has been shown to be toxic to the cells [52]. Cells deficient in HR or associated proteins, such as some members of the FA and BRCA families, as well as Rad18, demonstrate hypersensitivity to CPT [12, 24, 25, 27]]. Rad51 is a firmly established member of the HR repair pathway, and BRCA2 has been shown to directly bind and stabilize Rad51 on single-stranded DNA to facilitate HR
[43]. Interestingly, loss of either BRCA2 or FANCD2 was found to shift DSB repair away from HR and toward the more error prone NHEJ pathway. We and others have previously shown that E3 ubiquitin ligase Rad18 plays an important role in repair of fork-stalling lesions by promoting monoubiquitination of FANCD2 [12, 29, 30, 33, 34]. However, even though $\operatorname{Rad18,~} \operatorname{Rad} 51$, BRCA2, and FANCD2 are known to have roles in DSB repair, the interplay between these four proteins have not been thoroughly examined in the context of CPTinduced fork-stalling lesions. Here we show that Rad18 is important for the activation of FANCD2 (Figure $2 \mathrm{~A}$ ) and its nuclear foci formation (Figure 1 A and 1C) and deficiency in either Rad18 or FANCD2 decreases the protein levels and foci formation of BRCA2 and Rad51 in response to CPT (Figures 1B, 1C, 2A, 2B and

A
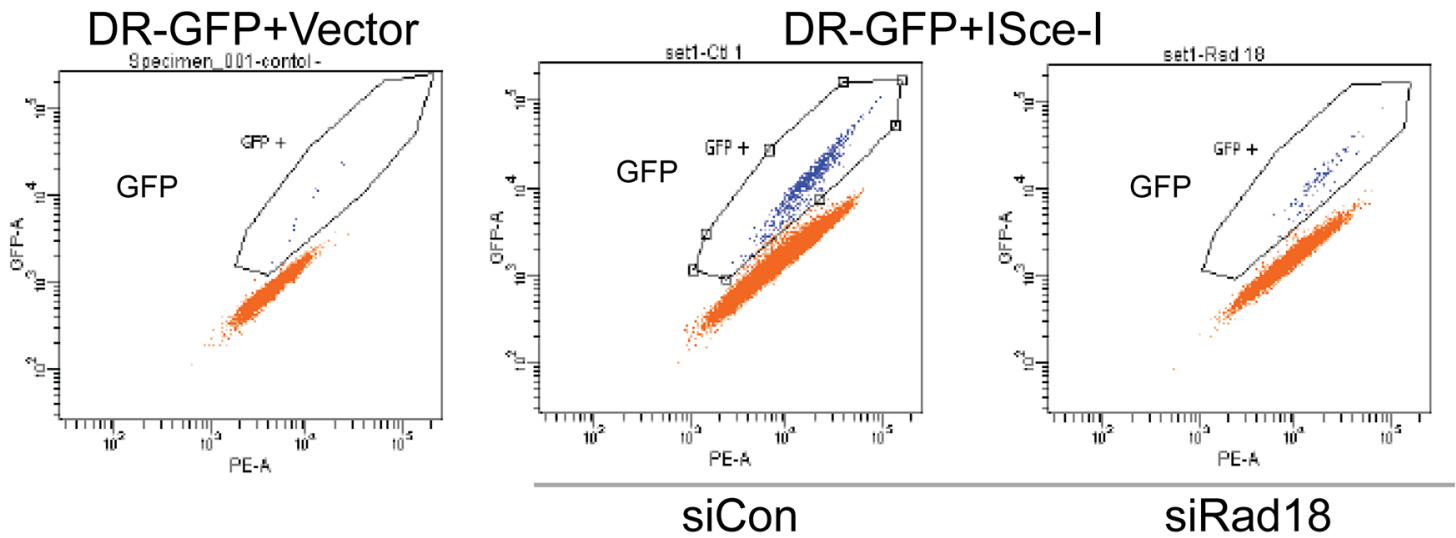

B
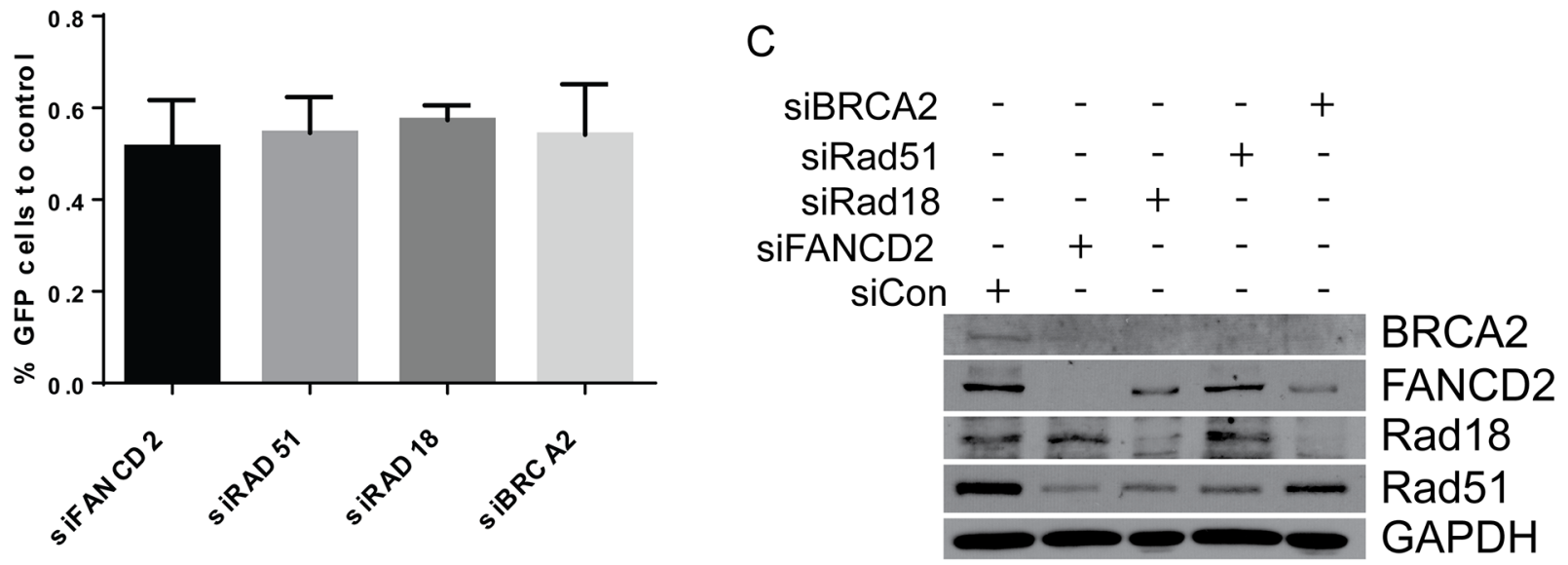

Figure 7: Downregulation of Rad18, FANCD2, BRCA2 and Rad51 results in similar levels of HR deficiency in ISce-I induced DSB repair. H1299 cells stably expressing the DR-GFP reporter were transiently transfected with siRNAs directed against Rad18, FANCD2, BRCA2 and Rad51, or a control siRNAs in addition to an expression vector for the restriction enzyme I-SceI or an empty vector. I-SceI causes a site specific DSB in the DR-GFP cassette, and repair of this DSB by HR allows GFP expression which can be quantitated by flow cytometry to measure HR. A. Representative flow analyses for GFP in cells without the I-SceI restriction enzyme ( $1^{\text {st }}$ panel), with fully function HR (control siRNA; panel 2), and after depletion of Rad18 (panel 3). B. Combined data from at least three separate experiments plotted as a percentage of control siRNAs GFP production (i.e., percentage of normal HR efficiency). The bars represent S.E.M. and all are statistically significant to control $(\mathrm{P}<0.05)$. C. Western blot from one representative experiment showing level of siRNA knockdown for each protein. 
A

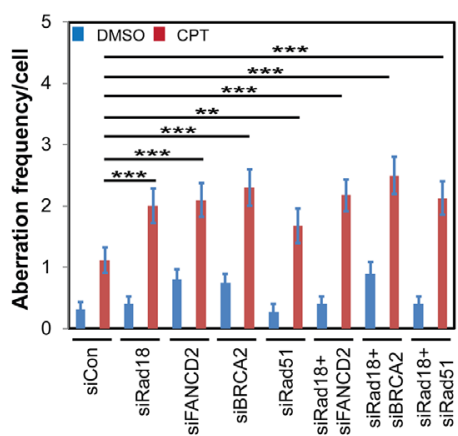

B

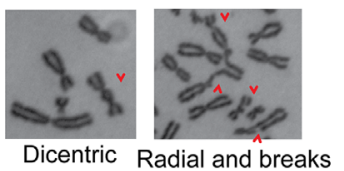

C
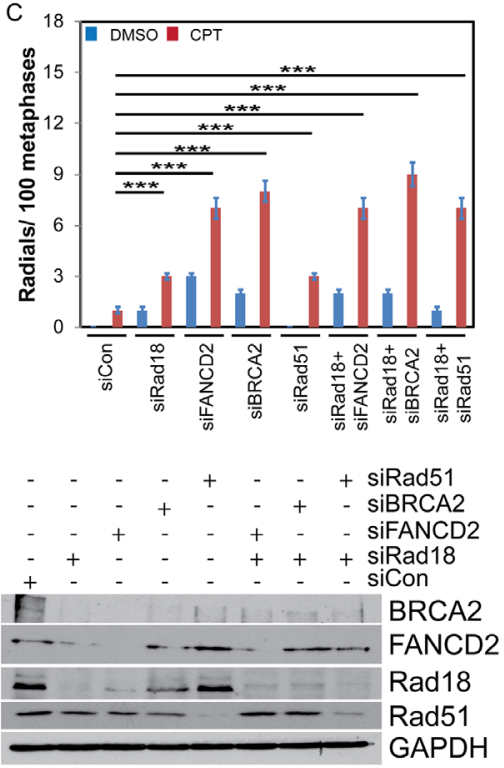

Figure 8: Knocking down Rad18, FANCD2, BRCA2 or Rad51 increases frequency of CPT-induced chromosomal aberrations (CA) and radials. H1299 cells were treated with siRNA to knock-down BRCA2, FANCD2, Rad18 or Rad51 singly or in combination with Rad18 or control siRNA. Cells were treated with vehicle (DMSO) or $500 \mathrm{nM} \mathrm{CPT}$ and colcemid for 2 hours, fixed and harvested for chromosome isolation. The chromosomes were deposited on a slide, stained and analyzed for the presence of aberrations and radial formation. A minimum of 50 metaphase spreads were analyzed. A. The frequency of aberrant chromosomes formed during CPT treatment in cells depleted for the indicated protein(s) normalized per cell. B. Representative images indicating the range of CA found under these conditions. A wider variety and more examples and data from different knockdowns can be found in Supplementary Figure S6. C. The number of radial chromosomes found in CPT-treated cells depleted for the protein(s) indicated. Both graphs (A) and (C) show the means from three independent experiments. Error bars represent the standard error of the mean. D. Western blot from one representative experiment showing levels of knockdown achieved. ** indicates statistical significance at $\mathrm{P}<0.05$ and $* * *$ indicates $\mathrm{P}<0.001$.

A)

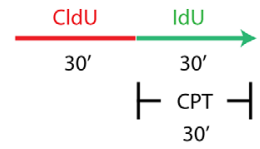

B)
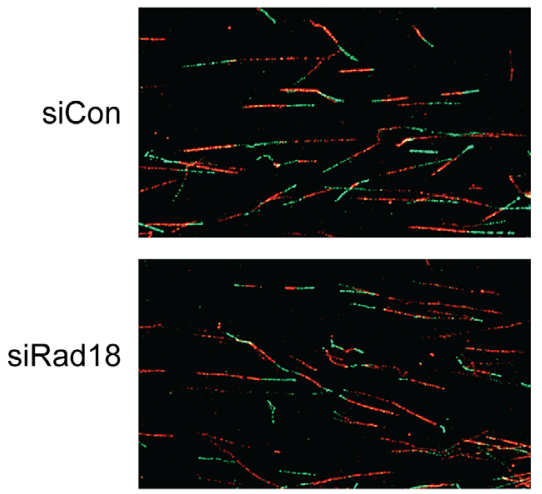

C)

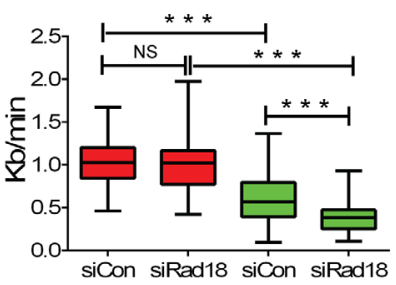

D)

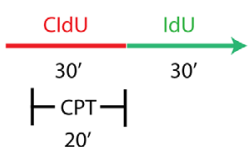

E)

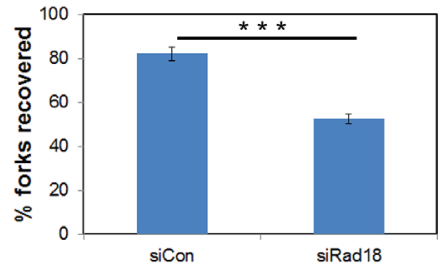

Figure 9: Rad18 is necessary for replication fork stability and recovery in response to CPT-induced replication blocks. H1299 cells treated with siControl or siRad18 were pulsed with thymidine analogues CldU and IdU and analyzed for replication fork stability A. and recovery D. Representative images of DNA fibers in siControl and siRad18 cells B. The measurements of obtained DNA fibers did not show any significant difference in the fork velocity ( $\mathrm{P}>0.05)$ between IdU-labelled and DMSO-treated siControl or siRad 18 cells $\mathbf{C}$. red bars). Whereas, upon treatment with CPT siRad18 cells showed a statistically significant $(\mathrm{P}<0.001)$ reduction in the fork velocity compared to siControl cells (C, green bars). Similarly, siRad18 cells treated with CPT also showed a statistically significant $(\mathrm{P}<0.001)$ decrease in the fork recovery compared to the siControl cells treated with CPT $\mathbf{E}$. Statistically significant $(\mathrm{P}<0.001)$ groups are indicated with $* * *$. NS indicates data not significant. 


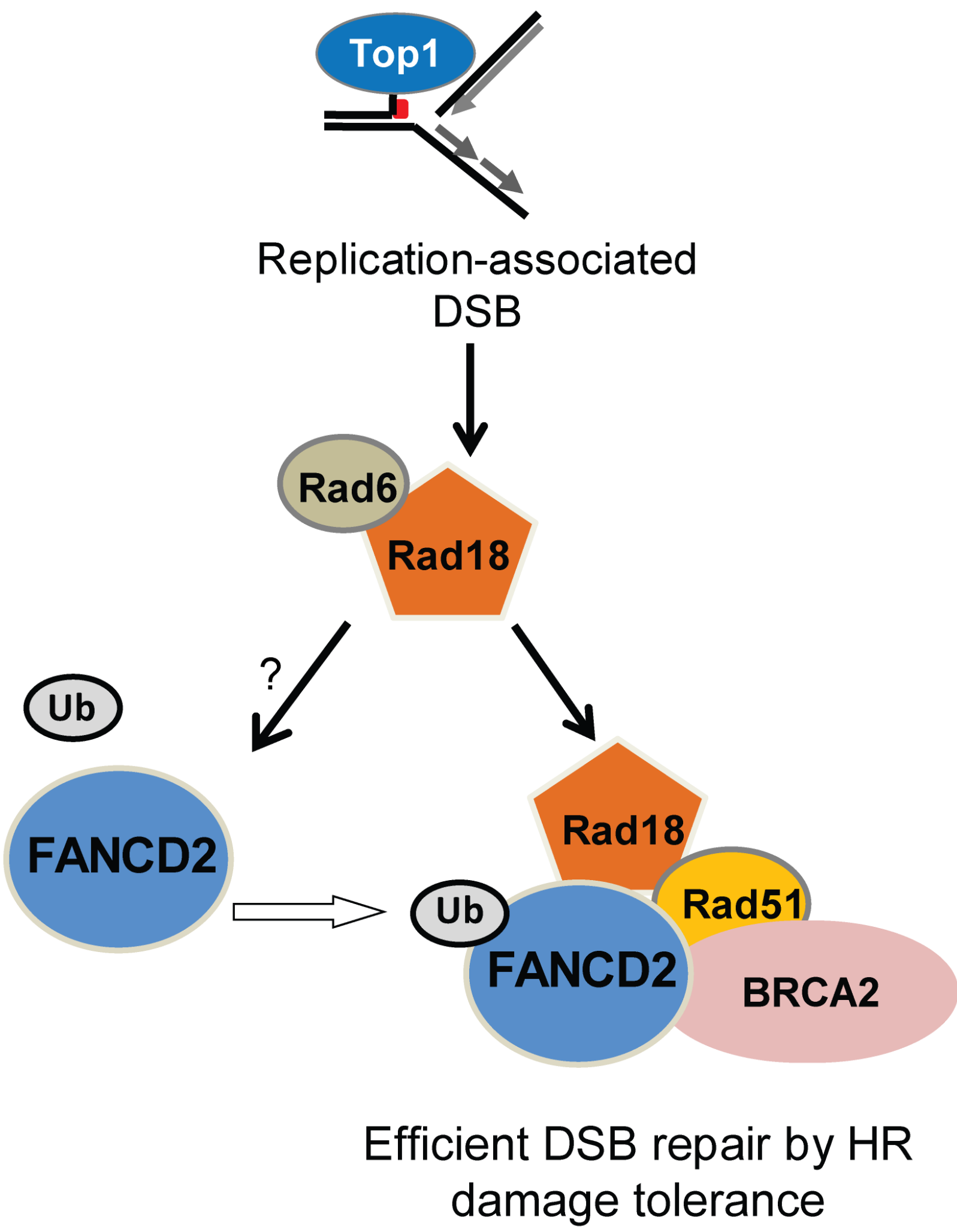

Figure 10: Summary of the data in a hypothetical model showing the functional relationships between Rad18, FANCD2, BRCA2 and Rad51 in repair of Top1-poisons-induced DSB. In this model Rad18 acts first in this repair complex and promotes activation of FANCD2. This allows monoubiquitinated FANCD2, BRCA2 and Rad18 to form a repair complex with Rad51 and facilitates efficient repair of CPT-induced DSB by HR. Loss of Rad18 diminishes the formation of this repair complex and Rad51-mediated repair of DSB by HR, which may lead to repair of replication-associated DSB by alternative repair pathways such as NHEJ causing chromosomal aberrations and cell death. 
Supplementary Figures S4B and S4D). On the other hand, BRCA2 downregulation only affected Rad51 foci (Figure 3 and Supplementary Figure S4B) and protein levels (Figure 4A), whereas Rad51 was not necessary for either stability (Figure 4B) or the ability of the other three to form CPT-induced foci (Figure 3 and Supplementary Figure S4). Similarly, downregulation of BRCA2 and Rad51 did not significantly impacted on monoubiquitination of FANCD2 in response to CPT (Figure 4A and 4B). Collectively, these results indicate that $\operatorname{Rad} 18$ acts upstream of FANCD2 by promoting its monoubiquitination, and together these proteins regulate BRCA2 and Rad51 to promote efficient repair of CPT-induced lesions by HR. More studies need to be done to determine the mechanism through which Rad18 regulates the stability of the other proteins.

The FA proteins (FANCs) are known to associate with replication forks and have been implicated to promote repair of stalled or collapsed forks by HR in association with BRCA proteins [36,39]. Previously, Rad18 has been shown to directly bind $\operatorname{Rad} 51 \mathrm{C}$, a paralog of $\operatorname{Rad} 51$ (also known as FANCO) and localizes it to sites of DSB to promote HR [26]. In this study downregulation of Rad18, FANCD2, BRCA2 and Rad51 resulted in similar deficiencies in HR assays. Moreover, Rad18 and FANCD2 status dependent foci formation of BRCA2 and Rad51 in response to CPT suggests a functional interaction between these four proteins. Rad18 dependent co-immunoprecipitation and co-localization of all four proteins in response to CPT supports existence of such interactions (Figures 5A, 5B and Supplementary Figure S6). However, further studies needed to establish the mechanistic basis for these interactions. It is unknown if these four proteins are interacting in one complex or multiple groups of two or three proteins, or if the interaction is due to association through other protein(s). Consistent with this, knocking down any of these genes alone or in combination with Rad18 resulted in similar sensitivities to CPT and gross chromosomal aberrations and radial chromosome formation (Figure 8 and Supplementary Figure S8), indicating a switch to more error prone repair mechanisms such as NHEJ causing chromosomal aberrations and cell death [33]. Additionally, Rad18 deficiency caused decreased replication fork instability and fork recovery in the presence of CPT (Figure 9), suggesting an important role for Rad18 in faithful DNA replication. In summary, these findings suggest a model (Figure 10), where Rad18 promotes monoubiquitination of FANCD2, and associates with a functional complex involving Rad51, BRCA2, and FANCD2 in the repair of CPT-induced stalled or collapsed forks.

\section{MATERIALS AND METHODS}

\section{Cell lines and reagents}

H1299 (human non-small cell lung carcinoma) and A2780 (human ovarian cancer) were obtained from
ATCC, and HCT116 and HCT116 Rad18 ${ }^{--}$(human colon cancer) cells were from Dr. Tadahiro Shiomi [27] and as used earlier [29]. Cells were cultured in Dulbecco's Modification of Eagle's Medium (DMEM) (Mediatech) supplemented with $10 \%$ fetal bovine serum (FBS) (Omega Scientific) and 1x Penicillin/Streptomycin sulfate (Gibco) [57]. Cells were routinely tested for mycoplasma contamination using Mycotest kit (Invitrogen) and were used prior to ten passages. CPT (Sigma) was used at concentrations and time periods indicated. Antibodies against the following targets were used: FANCD2, Rad51, 53BP1, GAPDH (all from Santa Cruz Biotechnology) BRCA2, RAD18 (Bethyl Laboratories) and $\gamma \mathrm{H} 2 \mathrm{AX}$ (Millipore). To express wild-type or the RING mutant Rad18, HCT116-/- cells were transfected with pcDNAmycRad18 and pcDNARad18 C28F, respectively.

\section{siRNAs and transfection}

To knock down expression of each gene, the following siRNA sequences were used: control AGUUACUCAGCCAAGAACGAUU, FANCD2 GCACCGUAUUCAAGUACAAUU, $\quad \operatorname{Rad} 18$ GAGCAUGGAUUAUCUAUUCAAUU and siRad183'UTR, 5'-UUA UAA AUG CCC AAG GAA AUU-3' [12], Rad51 UGUAGCAUAUGCUCGAGCGUU [58] and BRCA2 AACUGAGCAAGCCUCAGUCAAUU [59]. All siRNAs were purchased from Dharmacon. Transfections of siRNA oligonucleotides were done using Lipofectamine 2000 (Invitrogen) following the manufacturer's protocol.

\section{Clonogenic survival assays}

Cells were plated in triplicate in 6-well plates and treated overnight with indicated concentrations of CPT. Following drug treatment, cells were washed three times with PBS and three times with growth medium (CPT-free) to remove the drug, and allowed to form colonies. After 8 to 12 days, colonies were fixed in methanol and stained with crystal violet $(0.5 \% \mathrm{w} / \mathrm{v})$. Colonies containing more than 25 cells were counted either manually or using an automated imaging system (Gene Tools, Syngene), as described previously [60].

\section{Immunofluorescence}

For immunofluorescence, cells were seeded in triplicate into glass bottom, $35 \mathrm{~mm}$ culture dishes (FluoroDish - World Precision Instruments) or 6-well plates. After approximately 48 hours, cells were treated with CPT or vehicle control (DMSO) as indicated. Cells were fixed at room temperature with $4 \%$ formaldehyde for 10 minutes and then in cold $\left(-20^{\circ} \mathrm{C}\right) 100 \%$ methanol for 10 minutes. Fixed cells were then blocked in $10 \%$ goat serum for 30-60 minutes and washed 3 times with PBS. Cells were subsequently incubated overnight at $4^{\circ} \mathrm{C}$ with primary antibodies in PBS containing $5 \%$ BSA 
as described previously [57]. Cells were washed three or four times with PBS supplemented with 1\% BSA then incubated with appropriate fluorophore-conjugated secondary antibody (IgG-Cy3, IgG-FITC or IgG-Cy5 - Invitrogen) for $2 \mathrm{~h}$ at room temperature. Detection of protein foci was done using the Nikon Ti eclipse confocal microscope and images were acquired at 100x and as described previously [57].

\section{Western blot analysis}

Cells were grown in $10 \mathrm{~cm}$ cell culture dishes, transfected and treated as described above. Cells were washed in PBS and lysed in ice-cold cytoskeletal (CSK) buffer (10 mM PIPES (pH 6.8), $100 \mathrm{mM} \mathrm{NaCl}$, $300 \mathrm{mM}$ sucrose, $3 \mathrm{mM} \mathrm{MgCl} 2,1 \mathrm{mM}$ EGTA, $1 \mathrm{mM}$ dithiothreitol, $0.1 \mathrm{mM}$ ATP, $1 \mathrm{mM}$ Na3VO4, $10 \mathrm{mM}$ $\mathrm{NaF}$ and $0.1 \%$ Triton $\mathrm{X}-100$ ) freshly supplemented with protease and phosphatase inhibitors (Roche). Following determination of protein concentrations, gel samples were prepared in Laemmli buffer and heated to $100^{\circ} \mathrm{C}$ for fifteen minutes. Proteins were resolved by SDS-PAGE. Gels were electroblotted onto either a polyvinylidene fluoride (PVDF) or nitrocellulose membrane and blocked for 1 hour in 5\% milk powder dissolved in Tris-buffered saline containing $0.1 \%$ Tween-20 (TBST). Membranes were incubated with primary antibody for 2 hours, followed by incubation with an appropriate horseradish peroxidase-conjugated secondary antibody (Sigma) in TBST for 1 hour. Bound antibody was visualized using a chemiluminescence detection kit from Millipore, following manufacturer's instructions and detected on film (CL-Xposure, Thermo Scientific) [61].

\section{Immunoprecipitation}

Cells were grown and treated as detailed above, washed thrice in cold PBS and incubated with ice-cold RIPA buffer containing $20 \mathrm{mM}$ Tris. $\mathrm{HCl}, \mathrm{pH} 7.4,150$ $\mathrm{mM} \mathrm{NaCl}$, 1\% NP-40, 1 mM PMSF, 20 mM NaF, $1 \mathrm{mM}$ sodium vanadate and protease inhibitors for $30 \mathrm{~min}$ on ice. The cell lysates were centrifuged for $30 \mathrm{~min}$ at 30,000 g. These pre-cleared lysates were immunoprecipitated with specified primary antibody overnight, followed by incubation with protein A-Sepharose beads (Santa Cruz Biotechnology). Interacting proteins were eluted with $2 x$ Laemmli buffer and analyzed by Western blot [57].

\section{Metaphase spread detection and analysis}

Cells were grown and transfected with siRNA as described in previous sections. Cells were treated with vehicle (DMSO) or $500 \mathrm{nM} \mathrm{CPT} \mathrm{and} 0.1 \mu \mathrm{g} / \mathrm{ml}$ colcemid for 2 hours. Then, cells were harvested and placed in hypotonic solution $(0.075 \mathrm{M} \mathrm{KCl})$ for $20 \mathrm{~min}$ and subsequently fixed in Carnoy's fixative (methanol: acetic acid, 3:1) and deposited on microscope slides. Metaphase spreads were counted after fluorescence plus-Giemsa staining as described [57]. At least 50 metaphase spreads were analyzed for CA and radial formation and their mean \pm S.E. were calculated for each sample.

\section{Homologous recombination assay}

This method uses GFP reporter assay to measure HR activity and is described elsewhere [62]. Plasmids were obtained from Addgene. Human H1299 lung cancer cells were stably transfected with pDRGFP and selected for puromycin resistance $(10 \mu \mathrm{g} / \mathrm{ml})$. These stably transfected cells were grown to $60 \%$ confluency and transfected with a plasmid expressing the restriction enzyme I-SceI (pCBASce1). This restriction enzyme cuts the reporter plasmid and when repaired by HR GFP is expressed. GFP was measured by flow cytometry using a BD FACSCanto $^{\text {TM }}$ II (BD Biosciences).

\section{DNA fiber assay}

DNA fiber labeling analysis was used to assess DNA replication fork stability and fork recovery as described [63, 64]. In brief, for fork velocity, siControl and siRad18 H1299 cells were labelled with IdU for 30 mins followed by CldU for 30 mins with DMSO or CPT. For fork recovery, siControl and siRad18 H1299 cells were labelled with IdU for 30 mins with or without CPT in the last 20 mins followed by CldU for 30 mins. Cells were then harvested and resuspended in ice cold PBS. Then $2 \mu \mathrm{l}$ of the cell suspension was deposited over the slide and 10 $\mu l$ of lysis buffer $(0.5 \%$ SDS, $200 \mathrm{mM}$ Tris- $\mathrm{HCl}$ ph 7.4, 50 mM EDTA) was added. Slides were tilted to $15^{\circ}$ to stretch the DNA fibers, air dried, fixed in 3:1 methanol: acetic acid, denatured in 2.5M HCL and blocked with 5\% BSA in PBS. Then slides were incubated with anti-CldU Cy3 and anti-IdU Alexa Fluor 488 for an hour. More than 200 replication structures were measured for fork velocity and fork recovery, statistical analysis were performed using Prism 6 (GraphPad Software).

\section{Statistical analysis}

The Foci counting data and clonogenic survival data presented are averages of three independent experiments. Error bars represent the mean \pm S.D. All the data were analyzed either by using GraphPad Prism 6 or Excel 2010. The data presented in the manuscript are representative of three independent experiments unless otherwise mentioned.

\section{ACKNOWLEDGMENTS}

We thank Dr. Maria Jasin for kindly providing DRGFP reporter and I-SceI expression vectors. We also thank Steve McClellan for flow cytometry and Joel Andrews for help with confocal imaging. 


\section{FUNDING}

This work was supported by the National Institutes of Health [R01GM098956 to K.P.]; and the Abraham Mitchell Cancer Research Scholar Endowment grant. Funding for open access charge: National Institutes of Health [R01GM098956].

\section{CONFLICTS OF INTEREST}

The authors have no conflicts of interest to disclose.

\section{REFERENCES}

1. Wang JC. Cellular roles of DNA topoisomerases: a molecular perspective. Nat. Rev. Mol. Cell Biol. 2002; 3: 430-440.

2. Chen SH, Chan NL and Hsieh T. New mechanistic and functional insights into DNA topoisomerases. Annu. Rev. Biochem. 2013; 82: 139-170.

3. Pommier Y. DNA topoisomerase I inhibitors: chemistry, biology, and interfacial inhibition. Chem. Rev. 2009; 109: 2894-2902.

4. Pommier Y, Leo E, Zhang H and Marchand C. DNA topoisomerases and their poisoning by anticancer and antibacterial drugs. Chem. Biol. 2010; 17: 421-433.

5. Stewart L, Redinbo MR, Qiu X, Hol,WG. and Champoux JJ. A model for the mechanism of human topoisomerase I. Science. 1998; 279: 1534-1541.

6. Leppard JB. and Champoux JJ. Human DNA topoisomerase I: relaxation, roles, and damage control. Chromosoma. 2005; 114. 75-85.

7. Corbett KD. and Berger JM. Structure, molecular mechanisms, and evolutionary relationships in DNA topoisomerases. Annu. Rev. Biophys. Biomol. Struct. 2004; 33: 95-118.

8. Pommier,Y. Drugging topoisomerases: lessons and challenges. ACS Chem. Biol. 2013; 8: 82-95.

9. Koster DA, Palle, K, Bot ESM, Bjornsti MA. and Dekker NH. Antitumour drugs impede DNA uncoiling by topoisomerase I. Nature. 2007; 448. 213-217.

10. Staker BL, Hjerrild K, Feese MD, Behnke CA, Burgin AB. and Stewart L. The mechanism of topoisomerase I poisoning by a camptothecin analog. Proc. Natl. Acad. Sci. U. S. A. 2002 99. 15387-15392.

11. Regairaz M, Zhang YW, Fu H, Agama KK, Tata N, Agrawal S, Aladjem MI. and Pommier Y. Mus81-mediated DNA cleavage resolves replication forks stalled by topoisomerase I-DNA complexes. J. Cell Biol. 2011; 195: 739-749.

12. Palle K. and Vaziri C. Rad18 E3 ubiquitin ligase activity mediates Fanconi anemia pathway activation and cell survival following DNA Topoisomerase 1 inhibition. Cell Cycle Georget. Tex. 2011; 10: 1625-1638.
13. Pommier Y. Topoisomerase I inhibitors: camptothecins and beyond. Nat. Rev. Cancer. 2006; 6: 789-802.

14. Hsiang YH, Lihou MG. and Liu LF. Arrest of replication forks by drug-stabilized topoisomerase I-DNA cleavable complexes as a mechanism of cell killing by camptothecin. Cancer Res. 1989; 49: 5077-5082.

15. Tuduri S, Crabbé L, Conti $\mathrm{C}$, Tourrière H, Holtgreve-Grez H, Jauch A, Pantesco V, De Vos J, Thomas,A., Theillet,C., et al. Topoisomerase I suppresses genomic instability by preventing interference between replication and transcription. Nat. Cell Biol. 2009; 11: 1315-1324.

16. Shimura T, Torres MJ, Martin MM, Rao VA, Pommier Y, Katsura M, Miyagawa K. and Aladjem MI. Bloom's syndrome helicase and Mus81 are required to induce transient double-strand DNA breaks in response to DNA replication stress. J. Mol. Biol. 2008; 375: 1152-1164.

17. Holm C, Covey JM, Kerrigan D. and Pommier Y. Differential requirement of DNA replication for the cytotoxicity of DNA topoisomerase I and II inhibitors in Chinese hamster DC3F cells. Cancer Res. 1989; 49: 6365-6368.

18. Arnaudeau C, Lundin C. and Helleday T. DNA doublestrand breaks associated with replication forks are predominantly repaired by homologous recombination involving an exchange mechanism in mammalian cells. J. Mol. Biol. 2001; 307: 1235-1245.

19. Pommier Y., Barcelo JM, Rao VA, Sordet,O, Jobson AG, Thibaut L, Miao ZH, Seiler JA, Zhang H, Marchand, C, et al. Repair of topoisomerase I-mediated DNA damage. Prog. Nucleic Acid Res. Mol. Biol. 2006; 81: 179-229.

20. Pourquier P. and Pommier Y. Topoisomerase I-mediated DNA damage. Adv. Cancer Res. 2001; 80: 189-216.

21. Bjornsti MA. Cancer therapeutics in yeast. Cancer Cell. 2002; 2: 267-273.

22. Pommier Y. Redon C, Rao VA, Seiler JA, Sordet O, Takemura H, Antony S, Meng L, Liao Z, Kohlhagen G., et al. Repair of and checkpoint response to topoisomerase I-mediated DNA damage. Mutat. Res. 2003; 532: 173-203.

23. Adachi N, So S. and Koyama H. Loss of nonhomologous end joining confers camptothecin resistance in DT40 cells. Implications for the repair of topoisomerase I-mediated DNA damage. J. Biol. Chem. 2004; 279: 37343-37348.

24. Fedier A, Steiner RA, Schwarz VA, Lenherr L, Haller U. and Fink D. The effect of loss of Brcal on the sensitivity to anticancer agents in p53-deficient cells. Int. J. Oncol. 2003; 22: 1169-1173.

25. Singh TR, Bakker ST, Agarwal S, Jansen M, Grassman E, Godthelp BC, Ali AM, Du C, Rooimans MA., Fan Q., et al. Impaired FANCD2 monoubiquitination and hypersensitivity to camptothecin uniquely characterize Fanconi anemia complementation group M. Blood. 2009; 114: 174-180.

26. Huang J, Huen MSY, Kim H, Leung CCY, Glover JNM, $\mathrm{Yu}$ X. and Chen J. RAD18 transmits DNA damage signalling to elicit homologous recombination repair. Nat. Cell Biol. 2009; 11: 592-603. 
27. Shiomi N, Mori M, Tsuji H, Imai T, Inoue H, Tateishi S, Yamaizumi M. and Shiomi T. Human RAD18 is involved in S phase-specific single-strand break repair without PCNA monoubiquitination. Nucleic Acids Res. 2007; 35: e9.

28. Vaz F, Hanenberg H, Schuster B, Barker K, Wiek C, Erven V, Neveling K, Endt D, Kesterton I, Autore F., et al. Mutation of the RAD51C gene in a Fanconi anemia-like disorder. Nat. Genet. 2010; 42: 406-409.

29. Song IY, Palle K, Gurkar A, Tateishi S, Kupfer GM. and Vaziri C. Rad18-mediated translesion synthesis of bulky DNA adducts is coupled to activation of the Fanconi anemia DNA repair pathway. J. Biol. Chem. 2010; 285: 31525-31536.

30. Williams SA, Longerich S, Sung P, Vaziri C. and Kupfer GM. The E3 ubiquitin ligase RAD18 regulates ubiquitylation and chromatin loading of FANCD2 and FANCI. Blood. 2011; 117: 5078-5087.

31. Kannouche PL, Wing J. and Lehmann AR. Interaction of human DNA polymerase eta with monoubiquitinated PCNA: a possible mechanism for the polymerase switch in response to DNA damage. Mol. Cell. 2004; 14; 491-500.

32. Prakash S, Johnson RE. and Prakash L. Eukaryotic translesion synthesis DNA polymerases: specificity of structure and function. Annu. Rev. Biochem. 2005; 74: 317-353.

33. Kobayashi S, Kasaishi Y, Nakada S, Takagi T, Era S, Motegi A, Chiu RK, Takeda S. and Hirota K. Rad18 and Rnf8 facilitate homologous recombination by two distinct mechanisms, promoting Rad51 focus formation and suppressing the toxic effect of nonhomologous end joining. Oncogene. 2015; 13: 4403-11.

34. Park YE, Hayashi YK, Bonne G, Arimura T, Noguchi S, Nonaka I. and Nishino I. Autophagic degradation of nuclear components in mammalian cells. Autophagy. 2009; 5: 795-804.

35. Geng L, Huntoon CJ. and Karnitz LM. RAD18-mediated ubiquitination of PCNA activates the Fanconi anemia DNA repair network. J. Cell Biol. 2010; 191: 249-257.

36. Pickering A, Panneerselvam J, Zhang J, Zheng J, Zhang $\mathrm{Y}$. and Fei P. In vitro FANCD2 monoubiquitination by HHR6 and hRad18. Cell Cycle Georget. Tex. 2013; 12: 3448-3449.

37. Grompe M. and D'Andrea A. Fanconi anemia and DNA repair. Hum. Mol. Genet. 2001; 10: 2253-2259.

38. Park HK, Wang H, Zhang J, Datta S. and Fei P. Convergence of Rad6/Rad18 and Fanconi anemia tumor suppressor pathways upon DNA damage. PloS One. 2010; 5: e13313.

39. Garcia-Higuera I, Taniguchi T, Ganesan S, Meyn MS, Timmers C, Hejna J, Grompe M. and D'Andrea AD. Interaction of the Fanconi anemia proteins and BRCA1 in a common pathway. Mol. Cell. 2001; 7: 249-262.

40. Howlett NG, Taniguchi T, Olson S, Cox B, Waisfisz Q, De Die-Smulders C, Persky N, Grompe M, Joenje H, Pals G, et al. Biallelic inactivation of BRCA2 in Fanconi anemia. Science. 2002; 297: 606-609.

41. Sawyer SL, Tian L, Kähkönen M, Schwartzentruber J, Kircher M, University of Washington Centre for Mendelian Genomics, FORGE Canada Consortium, Majewski,J., Dyment,D.A., Innes,A.M., et al. Biallelic mutations in BRCA1 cause a new Fanconi anemia subtype. Cancer Discov. 2015; 5: 135-142.

42. Peng M, Xie J, Ucher A, Stavneze J. and Cantor SB. Crosstalk between BRCA-Fanconi anemia and mismatch repair pathways prevents MSH2-dependent aberrant DNA damage responses. EMBO J. 2014; 33: 1698-1712.

43. D'Andrea AD and Grompe M. The Fanconi anaemia/BRCA pathway. Nat. Rev. Cancer. 2003; 3:23-34.

44. Schlacher $\mathrm{K}, \mathrm{Wu} \mathrm{H}$. and Jasin MA. distinct replication fork protection pathway connects Fanconi anemia tumor suppressors to RAD51-BRCA1/2. Cancer Cell. 2012; 22: 106-116.

45. Jasin M. and Rothstein R. Repair of strand breaks by homologous recombination. Cold Spring Harb. Perspect. Biol. 2013; 5: a012740.

46. Petermann E, Orta ML, Issaeva N, Schultz N. and Helleday T. Hydroxyurea-stalled replication forks become progressively inactivated and require two different RAD51mediated pathways for restart and repair. Mol. Cell. 2010; 37: 492-502.

47. Wang X., Andreassen PR. and D'Andrea A.D. Functional interaction of monoubiquitinated FANCD2 and BRCA2/ FANCD1 in chromatin. Mol. Cell. Biol. 2004; 24:5850-5862.

48. Hussain S., Wilson J.B., Medhurst,A.L., Hejna,J., Witt,E., Ananth,S., Davies,A., Masson,J.-Y., Moses,R., West,S.C., et al. Direct interaction of FANCD2 with BRCA2 in DNA damage response pathways. Hum. Mol. Genet. 2004; 13: 1241-1248.

49. Venkitaraman AR. Tracing the network connecting BRCA and Fanconi anaemia proteins. Nat. Rev. Cancer. 2004; 4: 266-276.

50. Patel,K.J., Yu,V.P., Lee,H., Corcoran,A., Thistlethwaite, F.C., Evans,M.J., Colledge,W.H., Friedman,L.S., Ponder,B.A. and Venkitaraman,A.R. Involvement of Brca2 in DNA repair. Mol. Cell. 1998; 1: 347-357.

51. Oostra AB, Nieuwint AWM, Joenje H. and de Winter J.P. Diagnosis of fanconi anemia: chromosomal breakage analysis. Anemia. 2012; 2012: 238731.

52. Chapman JR, Taylor MRG. and Boulton SJ. Playing the end game: DNA double-strand break repair pathway choice. Mol. Cell. 2012; 47: 497-510.

53. Moynahan ME, Pierce AJ. and Jasin M. BRCA2 is required for homology-directed repair of chromosomal breaks. Mol. Cell. 2001; 7: 263-272.

54. Tutt A, Bertwistle D, Valentine J, Gabriel A, Swift S, Ross G, Griffin C, Thacker J. and Ashworth A. Mutation in Brca2 stimulates error-prone homology-directed repair 
of DNA double-strand breaks occurring between repeated sequences. EMBO J. 2001; 20: 4704-4716.

55. Pace P, Mosedale G, Hodskinson MR., Rosado IV, Sivasubramaniam M. and Patel KJ. Ku70 corrupts DNA repair in the absence of the Fanconi anemia pathway. Science. 2010; 329: 219-223.

56. Hashimoto Y, Chaudhuri AR, Lopes M. and Costanzo V. Rad51 protects nascent DNA from Mre11 dependent degradation and promotes continuous DNA synthesis. Nat. Struct. Mol. Biol. 2010; 17: 1305-1311.

57. Tripathi K, Mani C, Barnett R. Nalluri S, Bachaboina L, Rocconi RP, Athar M, Owen LB. and Palle K. Gli1 Regulates S-phase Checkpoint in Tumor Cells via Bid and its Inhibition Sensitizes to DNA Topoisomerase 1 Inhibitors. J. Biol. Chem. 2014; 10.1074/jbc.M114.606483.

58. Tsai M.-S., Kuo Y.-H., Chiu Y.-F., Su Y.-C. and Lin Y.-W. Down-regulation of Rad51 expression overcomes drug resistance to gemcitabine in human non-small-cell lung cancer cells. J. Pharmacol. Exp. Ther. 2010; 335, 830-840.

59. Fan S, Meng Q, Auborn K, Carter T. and Rosen EM. BRCA1 and BRCA2 as molecular targets for phytochemicals indole-3-carbinol and genistein in breast and prostate cancer cells. Br. J. Cancer.2006; 94: 407-426.
60. Tripathi K, Hussein UK, Anupalli R, Barnett,R, Bachaboina L, Scalici J, Rocconi RP, Owen LB, Piazza GA and Palle $\mathrm{K}$. Allyl isothiocyanate induces replication-associated DNA damage response in NSCLC cells and sensitizes to ionizing radiation. Oncotarget. 2015; 6: 5237-5252. doi: 10.18632/ oncotarget.3026.

61. Meng E, Mitra A, Tripathi K, Finan MA, Scalici J, McClellan S, Madeira da Silva L, Reed E, Shevde LA, PalleK., et al. ALDH1A1 maintains ovarian cancer stem cell-like properties by altered regulation of cell cycle checkpoint and DNA repair network signaling. PloS One. 2014; 9: e107142.

62. Pierce AJ, Johnson RD, Thompson LH. and Jasin M. XRCC3 promotes homology-directed repair of DNA damage in mammalian cells. Genes Dev. 1999; 13: 2633-2638.

63. Schwab RAV. and Niedzwiedz W. Visualization of DNA replication in the vertebrate model system DT40 using the DNA fiber technique. J. Vis. Exp. JoVE. 2011; $10.3791 / 3255$.

64. Thangavel S, BertiM., Levikova M, Pinto C, Gomathinayagam S, Vujanovic M, Zellweger R, Moore,H., Lee,E.H., Hendrickson,E.A., et al. DNA2 drives processing and restart of reversed replication forks in human cells. J. Cell Biol. 2015; 208: 545-562. 